\title{
Ligand Engineering of Titanium-Oxo Nanoclusters for Cerenkov Radiation-Reinforced Photo/Chemodynamic Tumor Therapy
}

Jingchao Li ${ }^{1, \#}$, Shuqi Dai ${ }^{2, \#}$, Ruixue Qin ${ }^{1}$, Changrong Shi ${ }^{1}$, Jiang Ming ${ }^{4}$, Xinying Zeng ${ }^{1}$, Xuejun Wen $^{l}$, Rongqiang Zhuang ${ }^{1}$, Xiaoyuan Chen ${ }^{3, *}$, Zhide Guo ${ }^{1, *}$, Xianzhong Zhang $^{1, *}$

${ }^{1}$ State Key Laboratory of Molecular Vaccinology and Molecular Diagnostics \& Center for Molecular Imaging and Translational Medicine, School of Public Health, Xiamen University

*E-mail: gzd666888@xmu.edu.cn; zhangxzh@xmu.edu.cn

${ }^{2}$ South China Advanced Institute for Soft Matter Science and Technology, School of Molecular Science and Engineering, South China University of Technology, Guangzhou 510640, People's Republic of China

${ }^{3}$ Yong Loo Lin School of Medicine and Faculty of Engineering, National University of Singapore, Singapore 117597, Singapore

*E-mail: chen.shawn@nus.edu.sg

${ }^{4}$ Department of Chemistry, Fudan University, Shanghai 200438, People's Republic of China 


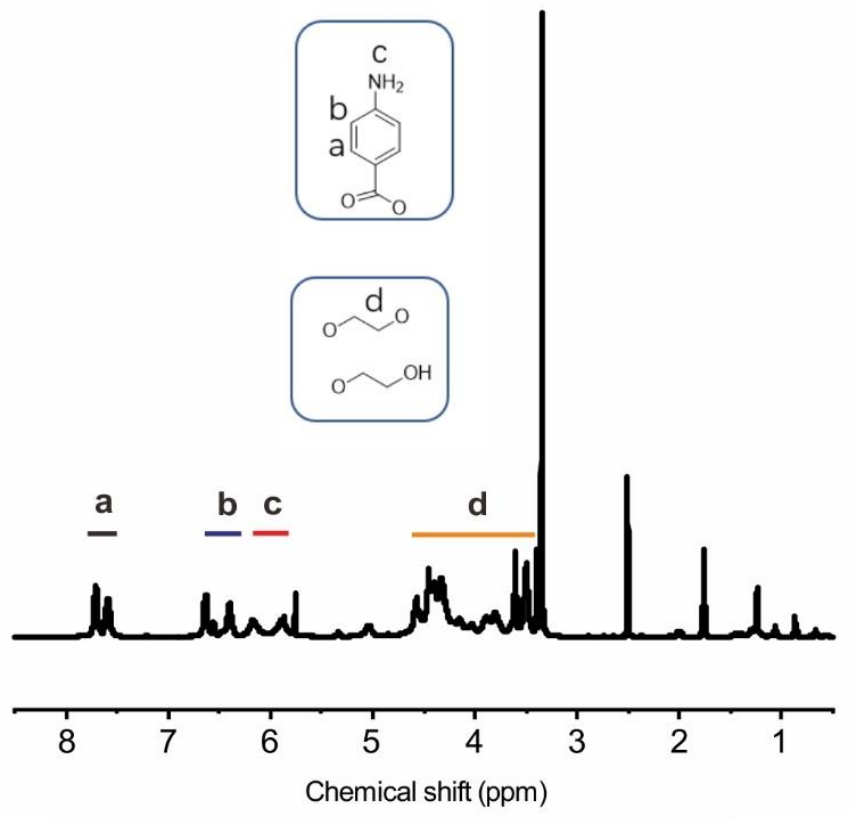

Figure $\mathrm{S} 1$. The ${ }^{1} \mathrm{H}$ NMR spectrum of NTOC. 
a)

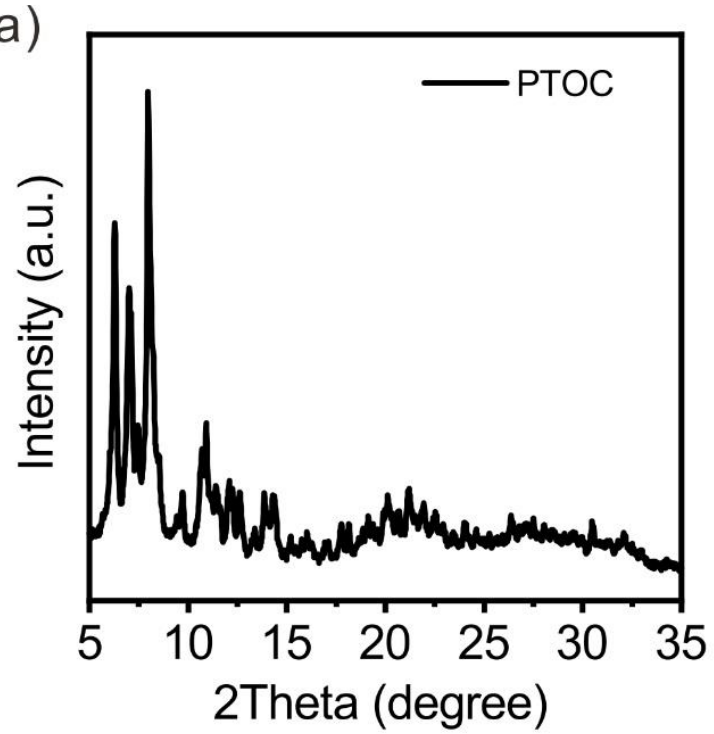

c)

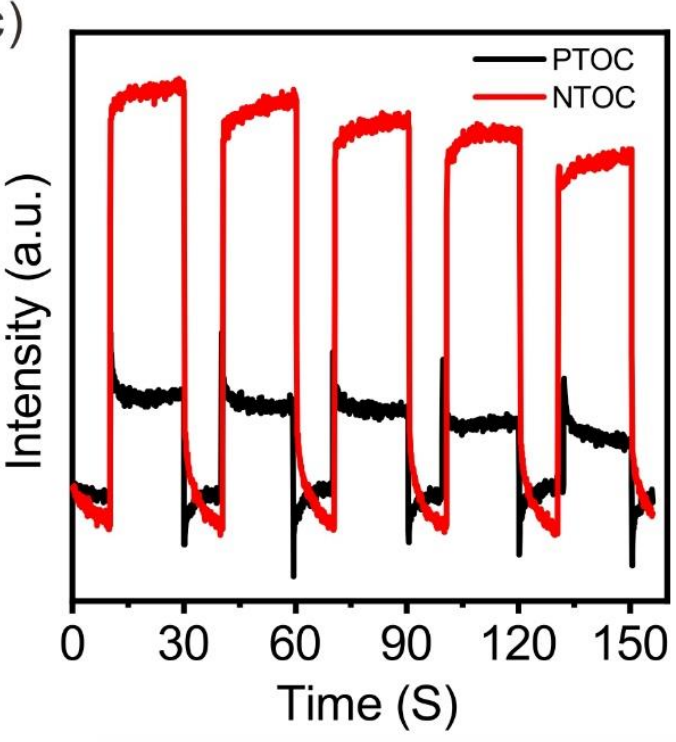

b)

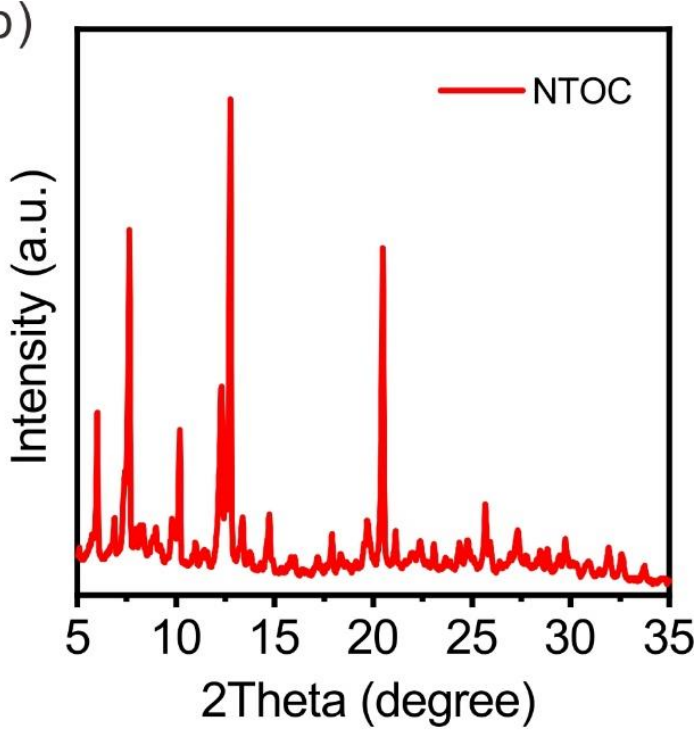

d)

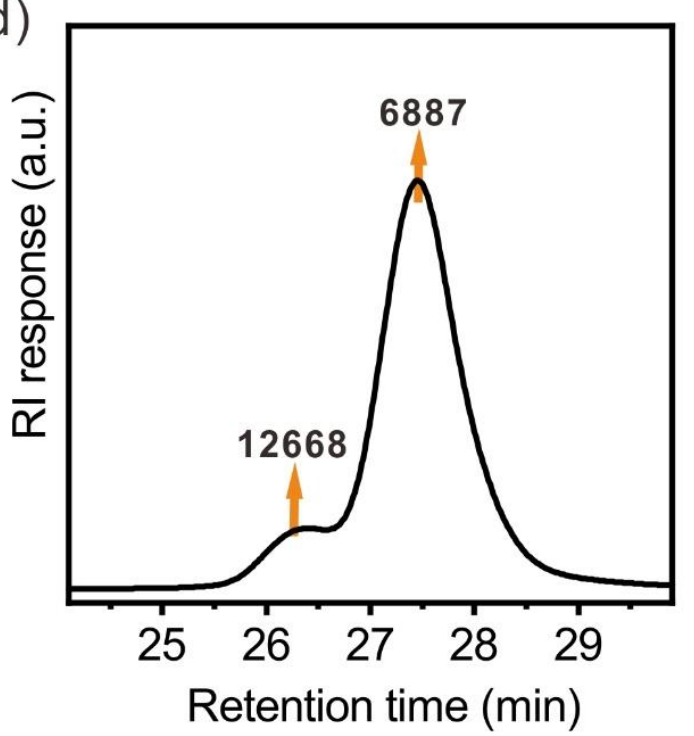

Figure S2. The XRD pattern of a) PTOC and b) NTOC. c) The photo-current signal of NTOC and PTOC. d) The GPC spectrum of TD. 


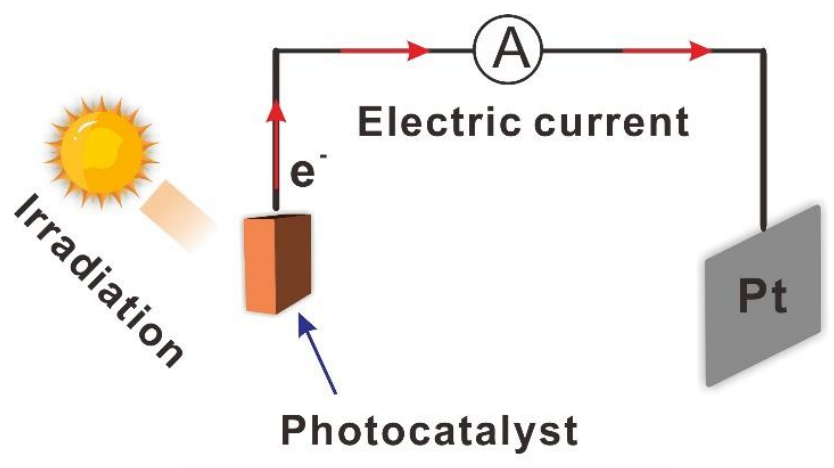

Figure S3. A schematic device for measuring the photo-current response signal of photocatalyst. 
a)

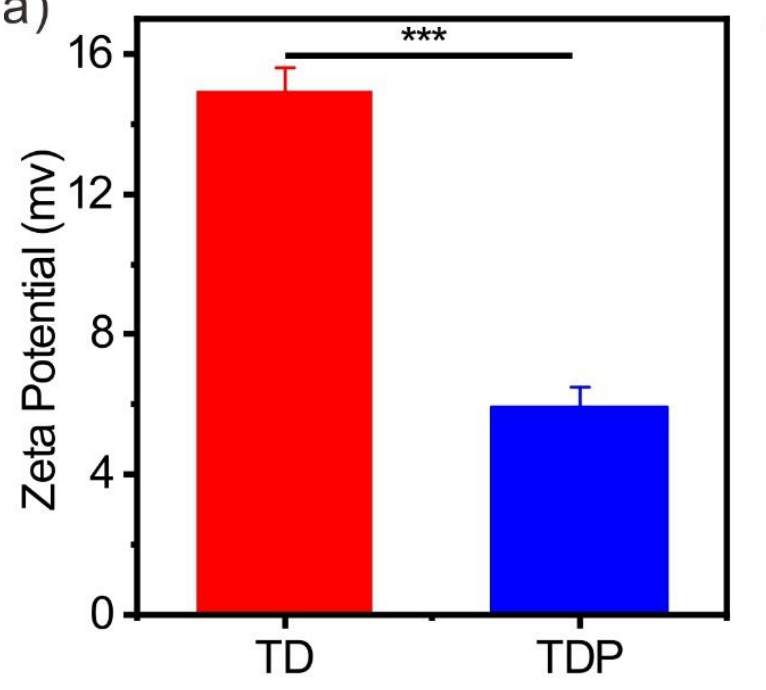

b)

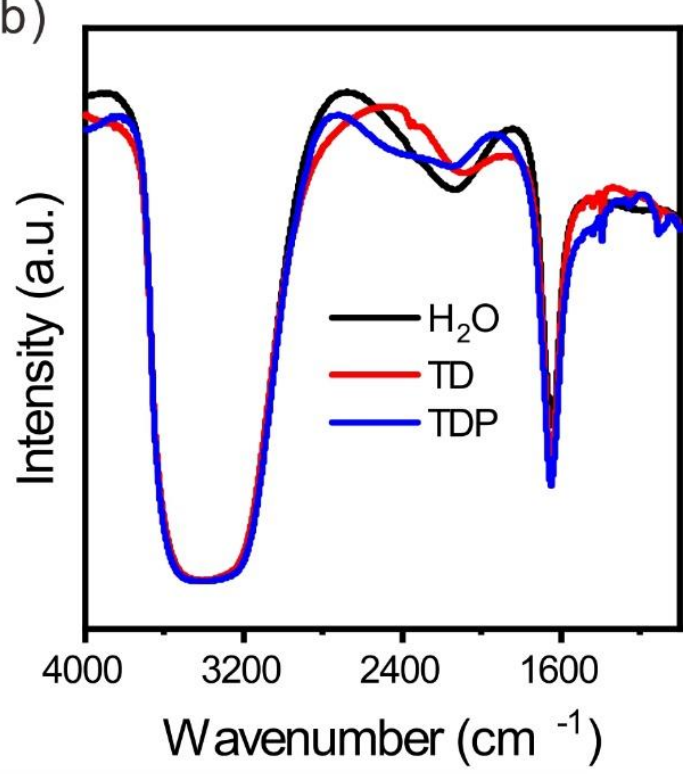

Figure S4. a) The surface Zeta-potential of TD and TDP. b) Fourier IR-spectrum of TD and TDP in aqueous solution. 


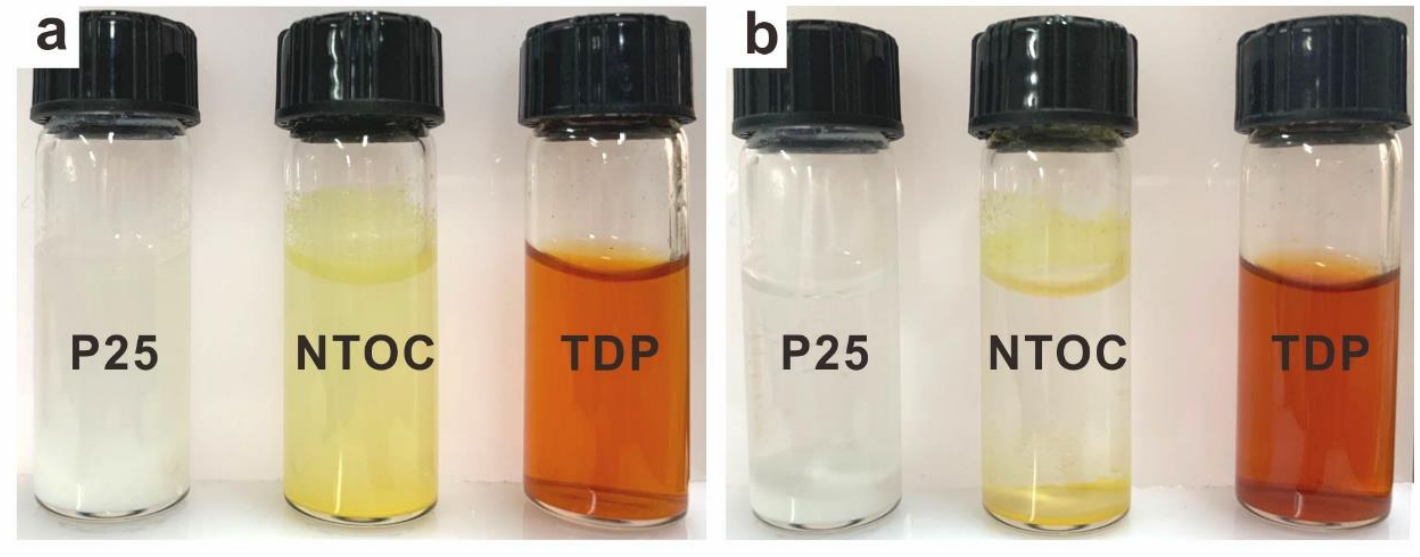

Figure S5. a) The images of saline solution of P25, NTOC and TDP, respectively. b)

The changes of solubility after standing for 2 days at room temperature. 
a)

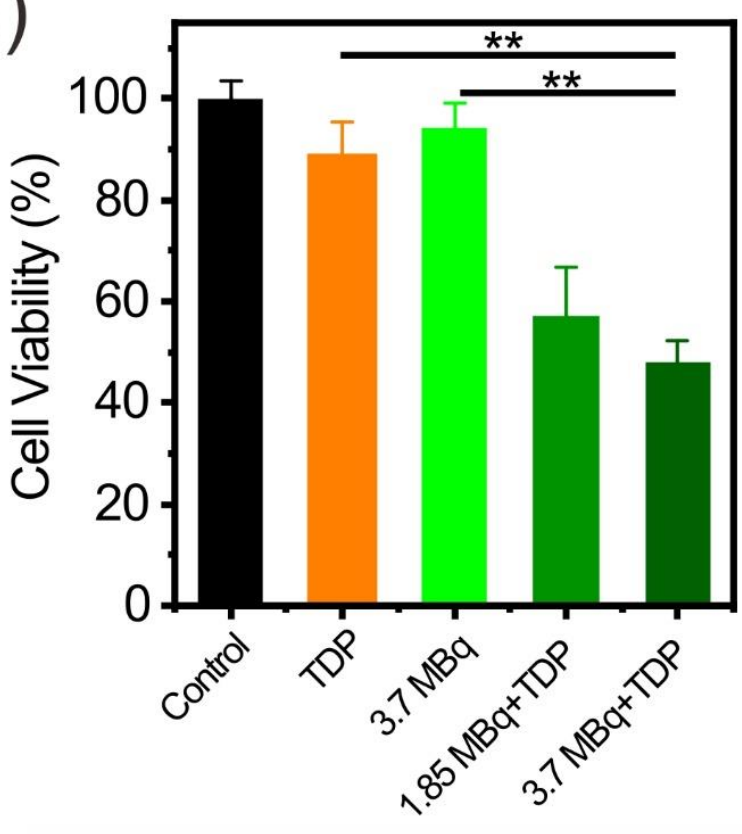

b)

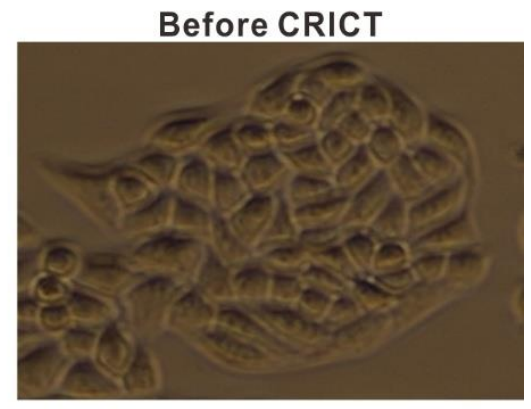

After CRICT

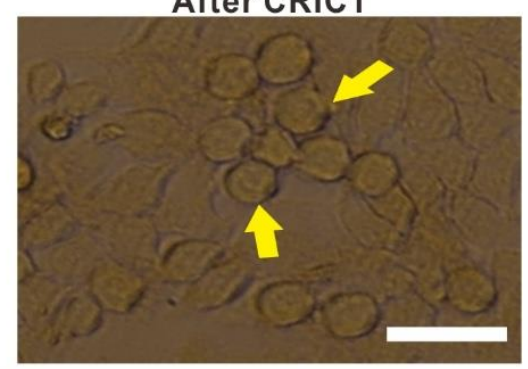

Figure S6. a) The therapeutic effect induced by ${ }^{131} \mathrm{I}$ at the cellular level. The cell viability was obtained by standard MTT assay. b) Microscopic images of HepG2 cells before and after CRICT (Scale bar: $40 \mu \mathrm{m}$ ). Yellow arrows point to the cancer cells with abnormal morphology. 

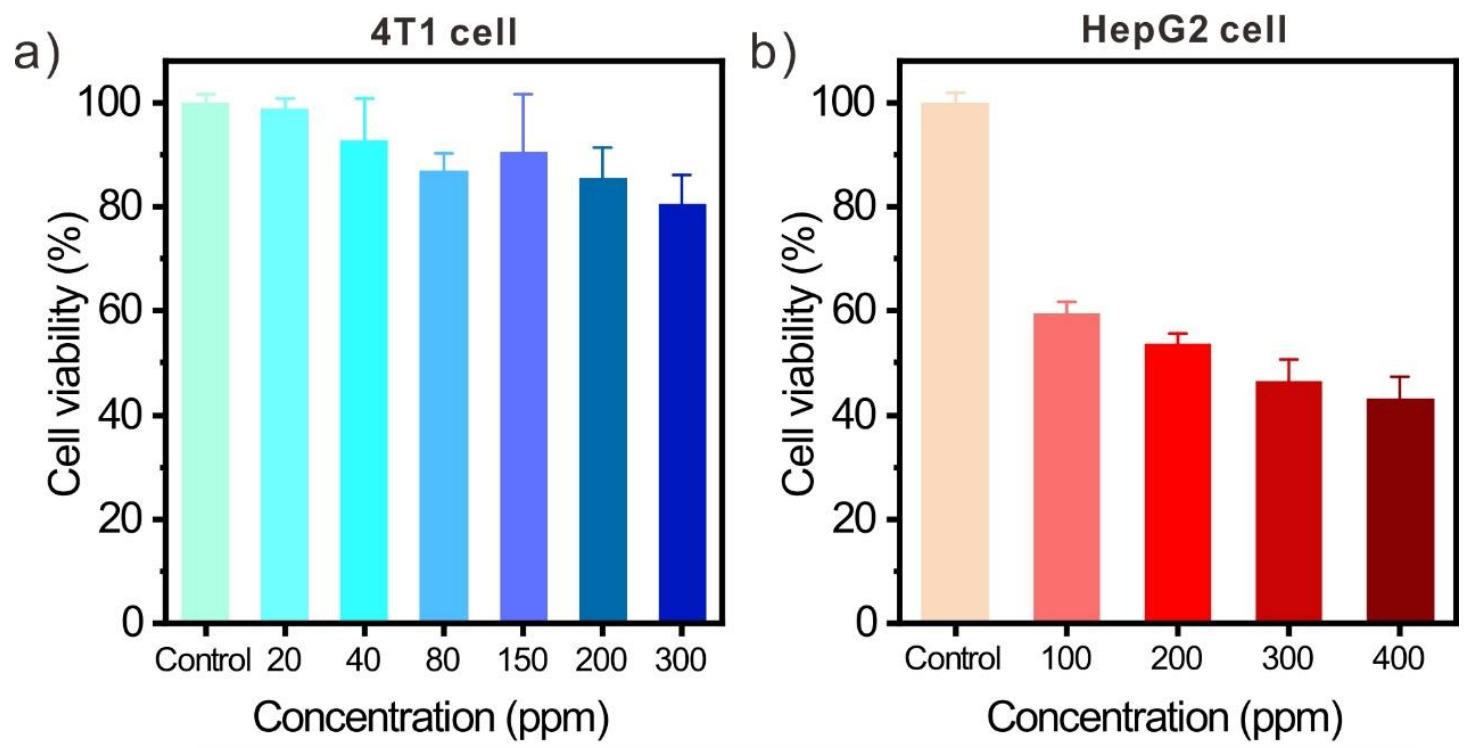

Figure S7. The distinct cytotoxicity of TDP on a) 4T1 and b) HepG2 cell lines. 


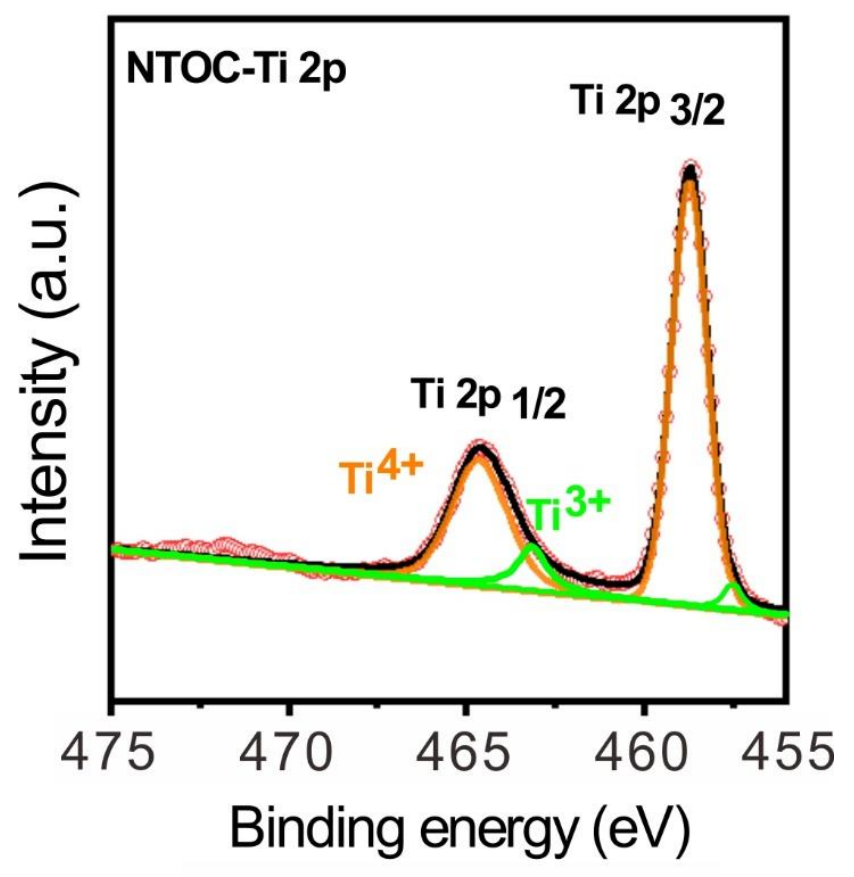

Figure S8. The XPS analysis of NTOC. 


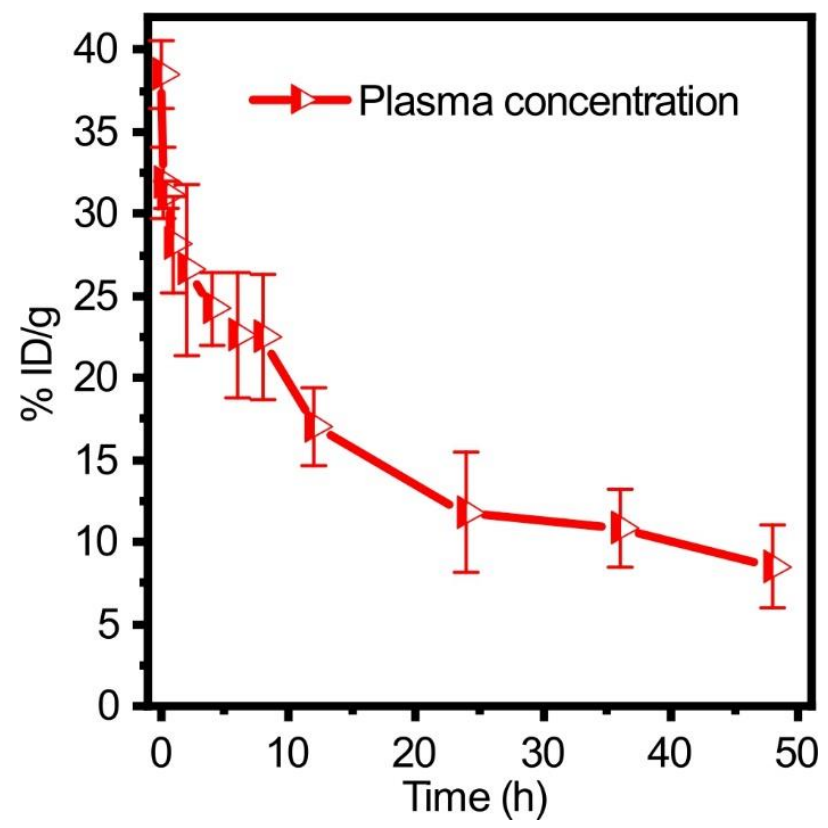

Figure S9. The blood circulation curve of i.v. injected TDP. 

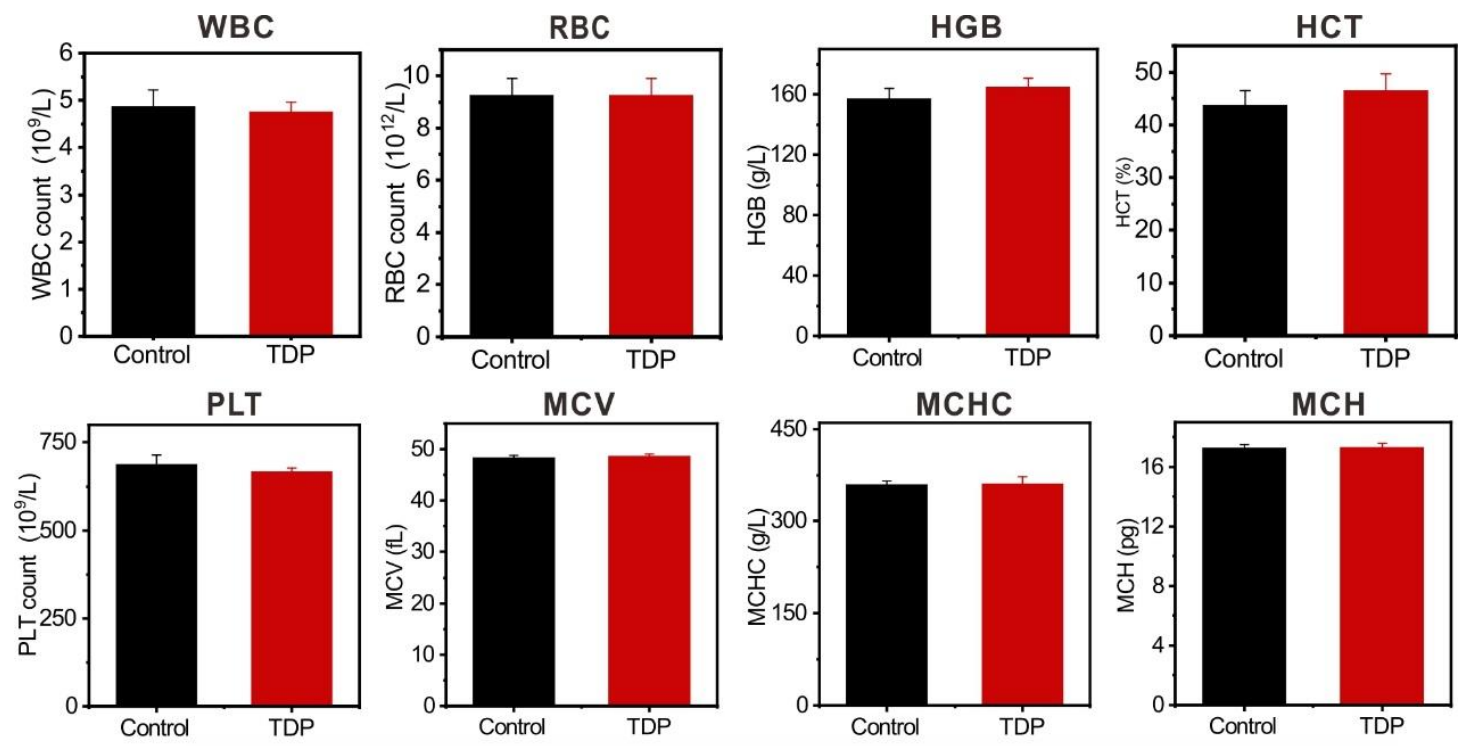

Figure S10. The blood routine examination at $48^{\text {th }} \mathrm{h}$ after i.v. administration of TDP. 

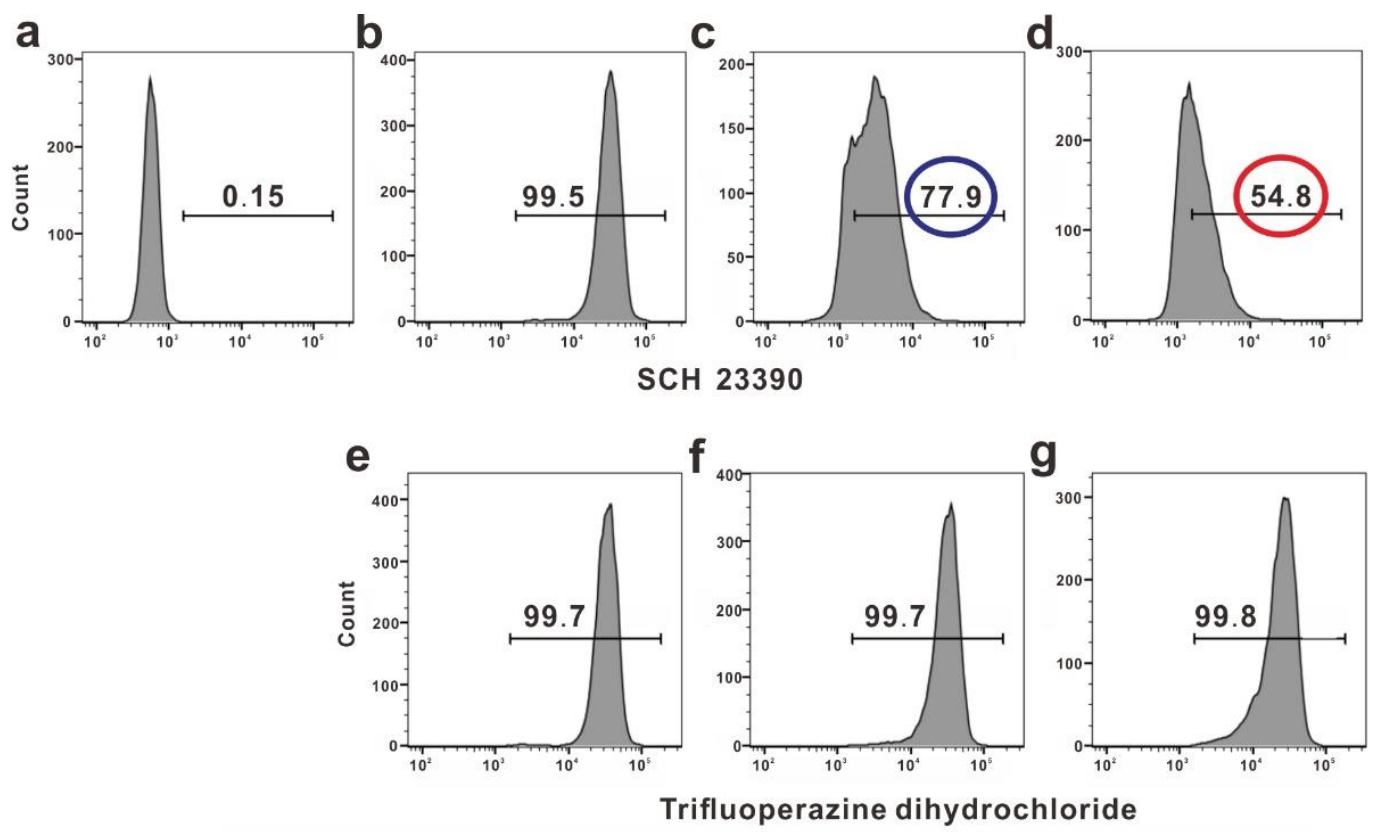

Figure S11. The cell uptake efficiency of TDP in different groups by flow cytometry. a) blank group, b) non-inhibited DRD1 and DRD2, c) the DRD1 was inhibited by SCH 23390, d) the dose of SCH 23390 doubled, e) blank group, f) the DRD2 was inhibited by Trifluoperazine dihydrochloride and g) the dose of Trifluoperazine dihydrochloride doubled. 


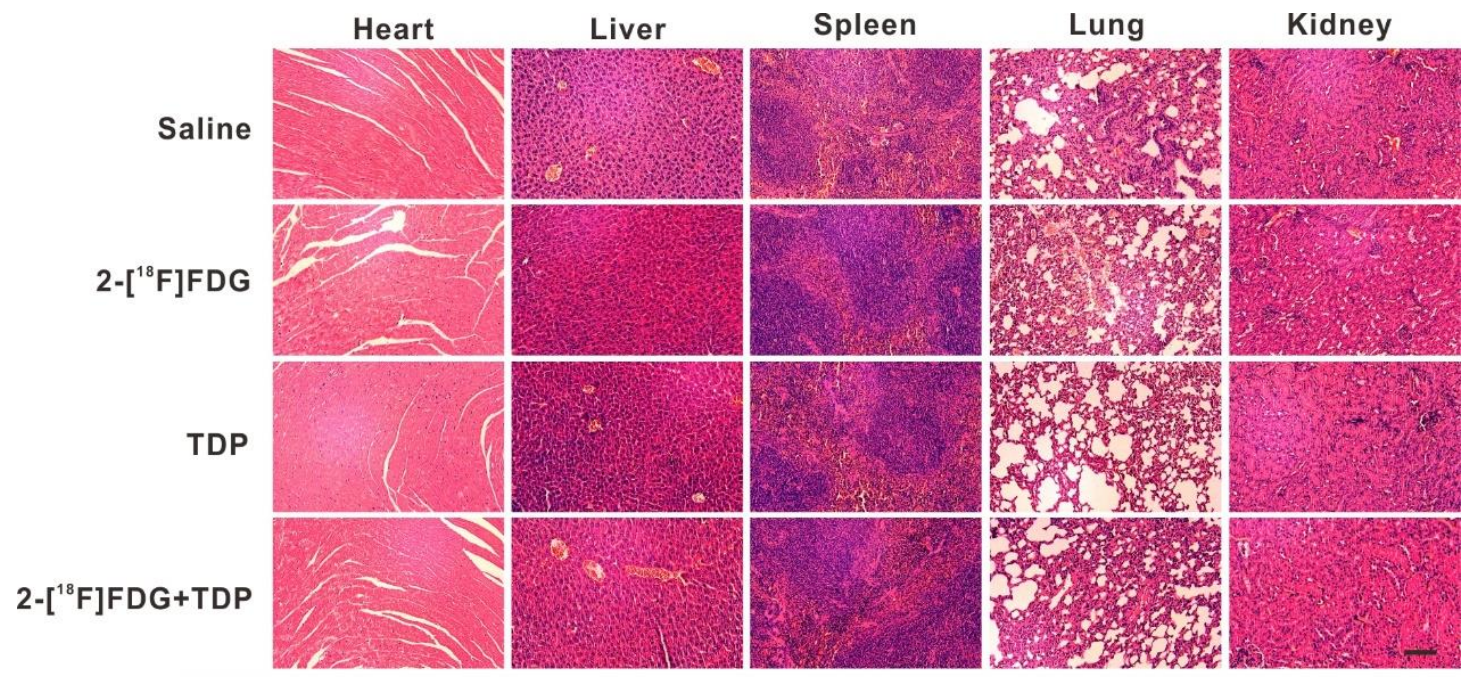

Figure S12. Representative histological H\&E staining of major organ tissues from each group (Scale bar: $50 \mu \mathrm{m})$. 


\begin{tabular}{ccc}
\hline & NTOC & TD \\
\hline C1s & $58.3 \%$ & $65.46 \%$ \\
\hline N1s & $4.4 \%$ & $5.78 \%$ \\
\hline Ti2p & $6.74 \%$ & $4.72 \%$ \\
\hline O1s & $30.56 \%$ & $24.32 \%$ \\
\hline
\end{tabular}

Table S1. The content of main element in NTOC and TD. 